\title{
Classification of tissue variations in X-ray scanning microdiffraction from thin sections of human brain \\ Abdullah Al Bashit ${ }^{1}$, Prakash Nepal ${ }^{2}$, Lee Makowski ${ }^{3}$ \\ ${ }^{1}$ Northeastern University ${ }^{2}$ Dept. of Bioengineering, ${ }^{3}$ Northeastern University bashit.a@northeastern.edu
}

$\beta$-amyloid plaques and neurofibrillary tangles (NFTs) are major hallmarks of pathology in Alzheimer's disease (AD). The plaques and NFTs exhibit wide structural variations in different regions of human brain. With the use of small (SAXS) and wide (WAXS) angle scattering from histological sections of AD human brain tissue, the underlying fibrillar shape, structure, and types of tissue lesions can be analyzed, detected, and classified over the different parts of individual and multiple human brains from AD subjects. Brain tissue from AD subjects is sectioned and scanned with the $5 \mu \mathrm{m}$ diameter X-ray beam to generate thousands of patterns arrayed across a region of interest. These data are used to generate maps of scattering attributes that reflect the variation of tissue structure and the spatial distribution of amyloid plaques and NFTs. However, the low intensity scattering from tissue lesions can be obscured by substrate, air, and mica scattering leading to low signal to noise ratio in the resulting diffraction patterns. The variation of tissue structure on multiple length scales combined with the polymorphism of NFT and amyloid aggregates presents significant challenges to structural characterization. We are addressing these challenges using state-of-art signal detection and classification methods including principal component analysis (PCA), support vector machine (SVM), and artificial neural network (ANN). The spatial distribution of structural attributes of $\beta$ amyloid and NFTs as mapped with scanning X-ray microdiffraction may lead to a better understanding of molecular basis of disease progression in $\mathrm{AD}$.

This work is supported by NIH grant number R21AG068972, 